\title{
A PERTINÊNCIA DE ADEQUAÇÃo DA GESTÃo CRIATIVA À IDENTIDADE DO INDIVÍDUO CRIATIVO
}

THE RELEVANCE TO ADAPT CREATIVE MANAGEMENT TO IDENTITY OF THE INDIVIDUAL CREATIVE

\section{LA IMPORTANCIA DE LA ADECUACIÓN ENTRE LA GESTIÓN CREATIVA Y} LA IDENTIDAD DEL INDIVIDUO CREATIVO

Henrique Muzzio

Doutor em Administração pela Fundação

Getúlio Vargas; Professor Adjunto da Universidade Federal de Pernambuco (UFPE) henrique.muzzio@ufpe.br

\section{Fernando Gomes de Paiva Júnior}

Doutor em Administração pela Universidade Federal de Minas Gerais; Professor Adjunto da UFPE

fernando.paivajr@gmail.com
Contextus

ISSNe 2178-9258

Organização: Comitê Científico Interinstitucional Editor Científico: Marcelle Colares Oliveira Avaliação : Double Blind Review pelo SEER/OJS Revisão: Gramatical, normativa e de formatação

Recebido em 19/03/2015

Aceito em 06/10/2015

$2^{\mathrm{a}}$ versão aceita em $24 / 11 / 2015$

$3^{\mathrm{a}}$ versão aceita em $02 / 12 / 2015$

\section{RESUMO}

O objetivo deste estudo é analisar a relação entre o indivíduo criativo e a gestão organizacional. Defende-se uma adequação de um modelo de gestão condizente com as nuances do universo organizacional da chamada economia criativa. Esse espaço simbólico de produção criativa se revela marcado por trabalhos precários. A demanda por afinação da identidade desse trabalhador criativo com a cultura das organizações pós-fordistas conduz a uma reflexão quanto às potencialidades de colaborações criativas com tecnologias de gestão centradas na busca por adequação das políticas de trabalho à nova lógica de mercado. Isso requer a formulação de sistemas de remuneração de incentivo às soluções inovadoras, disponibilização de horários flexíveis e ambiência organizacional aberta à criatividade. A partir de uma pesquisa bibliográfica, o texto faz uma proposição para a consolidação de uma gestão de organizações criativas alinhada com as características identitárias do indivíduo criativo nos níveis individual, grupal e cultural.

Palavras-chave: Economia criativa. Identidade. Gestão criativa. Inovação. Criatividade. 


\begin{abstract}
The objective of this theoretical essay is to analyze the relationship between the individual and creative management. Defends itself an adaptation of a consistent management model with the nuances of organizational universe called creative economy. This symbolic space for creative production is revealed to be characterized by precarious jobs. The demand for attuning the identity of the creative worker with the culture of post-Fordist organizations leads to reflections about the potential for creative collaborations with technologies centered on the search for work policies adequate to the new logic of the market. This requires the formulation of systems that provide incentive for innovative solutions and allow for flexible hours and the open work environments that nurture creativity. The text makes a proposition to consolidate management of creative organizations aligned with the identity characteristics of the creative individual in three levels: individual, group and cultural.
\end{abstract}

Keywords: Creative economy. Identity. Management technology. Innovation. Creativity.

\title{
RESUMEN
}

El objetivo de este estudio es analizar la relación entre el individuo creativo y la gestión organizacional. Se defiende una adaptación de un modelo de gestión coherente con los matices del universo organizacional de la llamada economía creativa. Este espacio simbólico de la producción creativa se reveló marcada por empleos precarios. La demanda por la afinación de la identidad de este trabajo creativo con la cultura de las organizaciones postfordistas conduce a una reflexión sobre el potencial de colaboraciones creativas con tecnologías de gestión centradas en la búsqueda de la adecuación de las políticas laborales a la nueva lógica del mercado. Esto requiere la formulación de la remuneración de fomento a las soluciones innovadoras, ofreciendo un horario flexible y entorno organizacional abierto a la creatividad. EL texto hace una propuesta para la consolidación de la gestión de las organizaciones creativas alineadas con las características identitarias de la persona creativa.

Palabras clave: Economía creativa. Identidad. Gestión creativa. Innovación. Creatividad.

\section{INTRODUÇÃO}

O objetivo deste ensaio teórico é analisar a relação entre o indivíduo criativo e a gestão. Defende-se uma adequação de um modelo de gestão condizente com as nuances do universo organizacional da chamada economia criativa. Tal debate justifica-se em função do esforço acadêmico para se compreender o modo como questões envolvendo o indivíduo, o elemento central da criação de valor, e as práticas de gestão se articulam em ambientes criativos, ainda que a atividade econômica relacionada à criatividade não seja algo novo. Embora já haja reflexão plausível no que tange à relação criatividade e inovação no cenário organizacional em setores diversos (ANDERSON; POTOČNIK; ZHOU, 2014), é notória a lacuna existente na literatura no que se refere ao indivíduo no contexto da organização criativa, quanto a uma possível afinação da identidade do trabalhador ao contexto instrumental aparentemente prevalecente no âmbito organizacional. 
A criatividade diz respeito à capacidade individual, associada a um contexto social, que proporciona rupturas conceituais ou práticas que culminam com a disponibilidade de novos processos, produtos, serviços ou conceitos para a aplicação comercial, isto é, seu efeito resultante tende a ser a inovação (ALVAREZ, 2010; ANDERSON; POTOČNIK; ZHOU, 2014; LOBATO, 2010; MARTENS, 2011). Nesse sentido, o indivíduo criativo é aquele profissional que, com suas competências, é capaz de contribuir com o desenvolvimento do processo inovador no cenário organizacional e em seu entorno por intermédio da operacionalização de projetos que viabilizam a efetivação dessa criatividade.

As colaborações criativas têm a capacidade de mudar o mercado, as organizações e os indivíduos (ALVAREZ, 2010, LOBATO, 2010), uma vez que os agentes portadores dessa criatividade possuem a aptidão para transformar desde a cultura do ambiente de trabalho, até as vivências dos grupos no interior das comunidades, reformulando os valores, as normas e a própria identidade (FLORIDA, 2011).

Diante da centralidade do indivíduo
SADLER-SMITH, 2012) e dado que a capacidade de criação e renovação dos artefatos pode contribuir para uma vantagem competitiva organizacional por meio de diferenciação (BARNEY, 1991), a identidade emerge como fator significativo para se compreender como as práticas gerenciais podem convergir para o perfil do trabalhador criativo. Portanto, pesquisas relacionadas à identidade e às relações de trabalho nesse campo tornam-se fontes de compreensão do meio em que emergem as novidades estruturadoras de novos formatos de gestão. Além do mais, são poucas as pesquisas que possuem como foco $\mathrm{o}$ trabalho na indústria criativa (ROWLANDS; HANDY, 2012).

Ao longo dos tempos, as inovações, as mudanças de paradigmas, os novos valores culturais vêm causando certa instabilidade na gestão organizacional. Portanto, o desconhecimento do novo, a resistência à mudança e o período de adaptação institucional são exemplos dos desafios gerenciais e organizacionais que emergem dos comportamentos de membros das equipes, à medida que vão sendo estabelecidas novas condições de trabalho no seu ambiente sociocultural (CLEGG; KORNBERGER; PITSIS, 2011).

na economia criativa (CHASTON; 
Esse cenário emergente gera elementos alternativos de análise no contexto organizacional, uma vez que o componente focal dessa discussão corresponde à identidade do trabalhador criativo e ao ambiente organizacional condizente com as expectativas mínimas de relações laborais emergentes desse profissional. A escolha da estratégia teórica, como metodologia da pesquisa, foi adotada em virtude da necessidade de fortalecimento epistemológico do estudo como forma consistente de se contribuir com estudos referentes à temática.

\section{UM BREVE PANORAMA DA ECONOMIA CRIATIVA}

A economia criativa é caracterizada, principalmente, pela composição no mercado de trabalho de indivíduos criativos, sendo uma fonte de vantagem competitiva e um mecanismo importante para crescimento nas economias pós-industriais. Os objetivos e as estratégias desses trabalhadores são retratados como impulsionados por motivos pessoais e internos, em vez de influenciados pelo contexto político e econômico em que trabalham (CHRISTOPHERSON, 2008). Assim, essa atividade está diretamente relacionada à capacidade, ao talento e à criatividade individual (CHASTON; SADLERSMITH, 2012; O’CONNOR; GU, 2010).

As mudanças econômicas estão alterando a estrutura do cotidiano social de forma duradoura nesta era atual, refletindo a emergência de uma sociedade em que o indivíduo criativo se encontra em ascensão, e, em decorrência disso, tem havido alterações no comportamento humano em relação ao sentido do trabalho. Com a evidência da classe criativa, essa perspectiva do trabalho deixa de ser marginal, chega ao centro da esfera econômica e, com isso, é possível se observar hoje indivíduos mais abertos a estilos de vida alternativos, em que se estabelece uma divisão crescente entre a classe criativa e as classes voltadas para atividades mais tradicionais (FLORIDA, 2011).

As atividades criativas são classificadas pela Conferência das Nações Unidas sobre Comércio e Desenvolvimento (UNCTAD) em quatro grandes grupos: patrimônio cultural, artes, mídia e criações funcionais (Quadro 1). Essa divisão consiste numa tentativa de dimensionar um amplo campo de atividades que podem ser mais bem compreendidas a partir de subdivisões. 
Quadro 1 - Classificação das indústrias criativas pela UNCTAD

\begin{tabular}{|c|c|c|}
\hline TEMA & DESCRIÇÃO & SUBGRUPOS \\
\hline Patrimônio Cultural: & \begin{tabular}{l}
\multicolumn{1}{c}{ É a herança que reúne } \\
aspectos culturais dos pontos de \\
vista histórico, antropológico, \\
étnico, estético e social.
\end{tabular} & $\begin{array}{l}\text { a) Expressões Culturais } \\
\text { Tradicionais: artesanato, festivais e } \\
\text { celebrações. } \\
\text { b) Sítios Culturais: sítios } \\
\text { arqueológicos, museus, bibliotecas e } \\
\text { exposições. }\end{array}$ \\
\hline Artes & $\begin{array}{l}\text { Inclui as indústrias } \\
\text { criativas baseadas puramente em } \\
\text { arte e cultura. }\end{array}$ & $\begin{array}{l}\text { a) Artes Visuais: pintura, } \\
\text { escultura, fotografia e antiguidades. } \\
\text { b) Artes Dramáticas: música } \\
\text { ao vivo, teatro, dança, ópera, circo e } \\
\text { marionetes. }\end{array}$ \\
\hline Mídia & $\begin{array}{l}\qquad \text { Finalidade de } \\
\text { comunicar para grandes } \\
\text { públicos: nessa e noutras } \\
\text { publicações; }\end{array}$ & $\begin{array}{l}\text { a) Publicação e mídia } \\
\text { impressa: livros, imprensa e outras } \\
\text { publicações. } \\
\text { b) Audiovisuais: cinema, } \\
\text { televisão, rádio e outras difusões. }\end{array}$ \\
\hline Criações Funcionais & $\begin{array}{l}\quad \text { São mais orientadas } \\
\text { pela demanda e serviços de } \\
\text { indústrias de bens e serviços } \\
\text { com finalidades funcionais. }\end{array}$ & $\begin{array}{l}\text { a) Design: interiores, moda, } \\
\text { gráfico, jóias; desenvolvimento (P\&D), } \\
\text { e serviços culturais. } \\
\text { b) Novas mídias: conteúdo } \\
\text { digital, software, jogos e animação. } \\
\text { c) Serviços de Criação: } \\
\text { arquitetura, propaganda, pesquisa e } \\
\text { desenvolvimento (P\&D), e serviços } \\
\text { culturais. }\end{array}$ \\
\hline
\end{tabular}

Fonte: UNCTAD (2010).

Mesmo com críticas em relação ao trabalho associado à dinâmica de desenvolvimento de tarefas criativas (PEUTER, 2011; SIEBERT; WILSON, 2013), pode-se dizer que, em geral, essas atividades fogem ao escopo de uma prática industrial tradicional em que, por exemplo, prevalece uma produção em massa com seus ganhos de produtividade associados, ou ainda, em que há uma elevada participação do trabalho mecanizado e uma padronização das tarefas dos indivíduos envolvidos. Nesse ambiente produtivo tradicional, o que se pode verificar é uma redução da importância do indivíduo em função da produção de bens vinculados a uma linha de montagem.
Esse fenômeno da economia criativa ocorre em esfera mundial, sendo vários os exemplos de regiões que se caracterizam como polos, os quais são: o Canadá (DAVIS; CREUTZBERG; ARTHURS, 2009), a Nova Zelândia (BOON; JONES; CURNOW, 2009), a Nigéria (LOBATO, 2010), a Austrália (GIBSON; LUCKMAN; WILLOUGHBYSMITH, 2010), o Reino Unido (O’CONNOR; GU, 2010), o Japão (SAAKI, 2010) a China (SONGJIE; XINGHUA, 2011), Curaçao (GOEDE; LOUISA, 2012) e o Brasil (GUERRA; PAIVA JR., 2013). Essas regiões incorporam a ideia de que as indústrias criativas são altamente visíveis porque 
exercem grande influência nos valores, nas atitudes e nos estilos de vida sociais (LAMPEL; LANT; SHAMSIE, 2000).

As amplitudes de atividades envolvidas com a criatividade, bem como seus exemplos empíricos, dão ideia da crescente importância dessa temática no desenvolvimento local. Como tais atividades requerem a maior participação do indivíduo na concepção e na produção dos bens e serviços, emerge uma necessidade de melhor compreensão do modo como as práticas gerenciais podem contribuir para que esse indivíduo amplie sua potencialidade criativa e permita uma melhor adequação de sua identidade com o contexto organizacional.

\section{ORGANIZAÇÕES E IDENTIDADES INDIVIDUAIS NO CENÁRIO CRIATIVO}

As organizações são complexos arranjos sociais criados com fins de ganho coletivo. Estruturas físicas, materiais, humanas e simbólicas são ordenadas em busca de objetivos mais amplos. Logo, para que esses arranjos possam ser bem sucedidos, as referidas estruturas precisam agir em harmonia. Diante do propósito deste estudo, prioriza-se a discussão no modelo de gestão e no indivíduo, em que debate-se a respeito da organização e sua interação com a temática da economia criativa.

\subsection{Gestão e o componente da criatividade}

A relação capital-trabalho no ciclo virtuoso fordista postula uma ampla divisão do trabalho com rígidos mecanismos de controle, relações trabalhistas autoritárias e paternalistas, equipamento especializado para levar a cabo uma produção massificada de bens padronizados. Isso levou esse cenário laboral intenso ser dominado pelo conflito capital-trabalho (HARDT; NEGRI, 2012).

Com o tempo, as fronteiras que antes delimitavam a organização burocrática tradicional foram sendo revisitadas, ao passo que as entidades se integraram e se descaracterizaram, passando a formar redes, cadeias, conglomerados e alianças estratégicas, a ponto de constituírem as denominadas organizações pós-fordistas (HARDT; NEGRI, 2012).

Em contraposição a uma perspectiva apologética do novo modelo de produção, surgem reflexões sobre sua eficácia na superação das incertezas e da saturação e instabilidade do mercado, ao ser levantada a questão de que, por mais flexível que seja a especialização, ela não pode evitar a força decrescente de uma redução da demanda por graus mínimos de relações de trabalho plausíveis do ponto de vista da qualidade de vida. 
Assim como o fordismo caracteriza-se por ser um tipo de organização da produção que envolve extensa mecanização e crescente divisão do trabalho, um novo padrão de gestão social e do trabalho poderia ser classificado em duas vertentes: a dos que defendem um neofordismo ou neotaylorismo e aqueles que apostam no chamado modelo pós-fordista, caracterizado como modelo flexível de gestão organizacional pela diferenciação integrada da organização da produção e do trabalho, pois conforme Tenório (2011, p. 1163), esse formato de organização do trabalho se pauta pela

trajetória de inovações tecnológicas em direção à democratização das relações sociais nas organizações. Concepção que contraria a fordista na medida em que esta se baseia na previsão de um mercado em crescimento, o que justificava o uso de equipamentos especializados a fim de obter economia de escala. Agora surgem equipamentos flexíveis cuja finalidade é atender a um mercado diferenciado, tanto em quantidade quanto em composição.

Apesar da impressão de dicotomia existente entre as duas concepções, observa-se na literatura a característica mais prevalente de uma repaginação da era do fordismo, assumindo traços das principais facetas do modelo anterior. Sob a égide dessa crítica, Costa (2015, p. 14) reconhece que tal modelo de produção não reverte as condições de qualidade de vida e liberdade do trabalhador, principalmente quando declara que

trata-se, pois, da busca cada vez mais
racionalizada pelo controle objetivo e
subjetivo do trabalhador, através da
tentativa de ocultação dos conflitos
estruturais entre capital e trabalho e
da tentativa, crescente, de
flexibilização do indivíduo conforme
os imperativos do amor ao trabalho,
da dedicação, da necessidade de
entrega, de auto-estima, etc. Sem defender uma visão evolucionista, pode-se dizer que a economia criativa representa, até então, um ápice dessa trajetória das organizações, a considerar que, segundo Bach (2013), as políticas de ciência, tecnologia e inovação têm sido profundamente renovadas nas últimas décadas, complementando ou substituindo abordagens heterodoxas em relação a linhas de argumentação obsoletas de gestão para solução das deficiências de mercado.

Essa linha de raciocínio pressupõe que, dado as características do mercado da economia criativa, da importância da inovação e da criatividade para suprir demandas de produtos não massificados, ou ainda, da condição dos produtos ou dos serviços que carregam um alto grau de 
trabalho analítico e criativo, o modelo gerencial dessas organizações não deveria estar pautado em pressupostos da economia tradicional fordista.

Nesse modelo gerencial criativo, seria mais adequado se os sistemas de controle, de remuneração, de liderança, de avaliação de desempenho, enfim, todas as práticas de gestão, estivessem adaptadas a uma nova realidade, em que o indivíduo assume um papel protagonista nesse tipo de produção atrelada ao trabalho intensivo em conhecimento.

\subsection{A Identidade do indivíduo criativo e o contexto sócio-organizacional}

A identidade pode ser analisada por diferentes níveis. No plano individual, no qual o indivíduo estabelece uma relação com sua própria condição de ser humano, essa identidade, oriunda de uma condição psicológica, produz significados para esse indivíduo estabelecer relações consigo e com o mundo que o cerca. As percepções desse plano no contexto do trabalho podem ser exploradas no que diz respeito ao conteúdo do trabalho ou da gestão de carreira.

O segundo nível ocorre no plano organizacional, no qual determinadas características coletivas são vistas como centrais e definidoras da organização (CORLEY; GIOIA, 2004). Nesse nível, o indivíduo estabelece as conexões com seu grupo social e busca um equilíbrio entre o que ele deseja ser e o que o grupo espera que ele seja, isto é, mesmo sendo originalmente um fator individual, a identidade é referenciada (e legitimada) no coletivo (BROWN; TOYOKI, 2013).

O terceiro nível se estabelece no plano institucional, em que o ambiente sociopolítico dos profissionais representa fonte material de recursos interpretativos e de legitimidade, esse ambiente tanto habilita como restringe a reconstrução da identidade do papel profissional (CHREIM; WILLIAMS; HININGS, 2007). A identidade é uma condição mutável, muitas vezes até antagônica, em que complexas variáveis dão um tom fluido e instável em decorrência dos fatos sociais (ALVESSON, 2010; CLARKE; BROWN; HAILEY, 2009).

Machado (2003) faz uma divisão em que a identidade ocorre nas dimensões pessoal, social, laboral e organizacional. Cada um desses níveis possui diferenciações quanto a objetos de estudo, meios de construção, períodos de ocorrência, espaços de construção e finalidades. Especificamente sobre o plano organizacional, a autora destaca a identidade vinculada à organização na qual o indivíduo incorpora as instruções do imaginário para sua orientação no plano organizacional. 
A ênfase dada neste estudo contempla a identidade no plano organizacional, ou seja, ainda que o indivíduo tenha sua identidade pessoal, ela não está isolada do contexto da organização. Como a identidade é um processo social, ela corresponde, ao mesmo tempo, tanto ao que é projetado como ao que é percebido, e; portanto, a temática se revela como sendo uma facticidade processual construída em algum lugar entre o comunicador e seu público-alvo (YBEMA ET AL., 2009).

A relação de identidade organizacional explora como os atores organizacionais mostram o que eles são uns aos outros e a eles mesmos (WANG; CHENG, 2010), bem como demonstra como diferentes formas discursivas - tais como a autobiografia, a narrativa, e as interações cotidianas - podem ilustrar a experiência de agentes individuais em moldar, reconstruir e serem sujeitos em suas realidades (YBEMA ET AL., 2009). Essa identidade de trabalho é constituída dentro de regimes discursivos de base organizacional que oferecem posições, ou espaços epistemológicos, para a ocupação de indivíduos e de grupos (CLARKE; BROWN; HAILEY, 2009).

A identidade, portanto, compreende algo como autonarração reflexiva que se inspira, por um lado, nas narrativas e nos discursos socialmente fornecidos e, por outro, na interação face a face de performances dramatúrgicas (DOWN; REVELEY, 2009). Isso ocorre em função de a identidade do trabalho se referir aos processos mutuamente constitutivos pelos quais as pessoas se esforçam para formar noções relativamente coerentes e distintas de seus interesses (BROWN; TOYOKI, 2013).

No que diz respeito à identidade na esfera da economia criativa, esta compreende as atividades econômicas em que atributos simbólicos e estéticos se inserem no cerne da criação de valor (DEFILLIPPI; GRABHER; JONES, 2007). As identidades estão em deslocamento num momento de reestruturação e mudança da sociedade, fato que ilustra a emergência do fenômeno criativo se projetando no cenário global (BURGEN-HELMCHEN， 2013). A identidade, nesse sentido, aparece como logro de produções criativas, a exemplo do que ocorre no campo do audiovisual, ilustrado no caso do cinema produzido no estado de Pernambuco (GUERRA; PAIVA JR, 2013).

O trabalhador criativo pode exercer sua atividade tanto de maneira autônoma como por contrato formal de trabalho em uma organização, que corresponde à perspectiva na qual o artigo irá se deter com respeito ao debate identitário. Para o trabalhador criativo vinculado formalmente 
a uma organização, várias perspectivas identitárias podem emergir no universo organizacional. Uma delas está relacionada ao modo como esse indivíduo interpreta sua condição no âmbito de um arcabouço organizacional dotado de práticas e regras burocráticas não condizentes com sua identidade. Em regra, pessoas extremamente criativas trabalham em uma equipe relativamente pequena, conduzida por indivíduos altamente motivados, criada em torno de princípios flexíveis e de autoorganização. Nesses espaços, aflora a criatividade, que engloba a capacidade de combinar dimensões de experiências anteriormente não relacionadas (CLEGG; KORNBERGER; PITSIS, 2011). Mas há nas organizações de estruturas padronizadas algum ambiente benigno que seja condizente com a identidade do indivíduo criativo?

Como já destacado, na atividade criativa, os indivíduos têm papel preponderante em comparação com a economia tradicional (BENDASSOLLI; BORGES-ANDRADE, 2011), explorando sua capacidade transformadora na busca pelo uso de plataformas tecnológicas em direção à aplicação de soluções inovadoras nos aparatos gerados pela integração de ciência, tecnologia e arte. Tal criatividade diz respeito à habilidade de se produzir trabalho que se constitui uma novidade e, ao mesmo tempo, é valiosa para lidar com o advento da economia do conhecimento.

Para que a identidade individual e a organizacional desse indivíduo criativo possam ser dotadas de sentido, fatores de influência da gestão organizacional deveriam estar condizentes com essas identidades individuais. Exemplos desses fatores são: a cultura organizacional, o estilo de liderança, a política de valorização de pessoal, dentre outros. Notadamente, deveria haver uma permissividade organizacional para lidar com um indivíduo mais autônomo, mais independente, mais consciente de sua relevância no processo organizacional, mais vinculado com as condições sociopolíticas internas e externas, mais sabedor de suas limitações e potencialidades, mais autodirigido, dentre outros. Mas as organizações do setor criativo estão estruturadas para esse indivíduo?

\section{REFLETINDO SOBRE A PERTINÊNCIA DE ADEQUAÇÃO ENTRE GESTÃO E O INDIVÍDUO CRIATIVO}

Os elementos de gestão são usados como meios para alcançar os objetivos organizacionais. Ao se considerar a relevância das pessoas na concepção, na condução e no propósito organizacional, mesmo com seu viés instrumental, não 
seria difícil supor que a cultura, as regras, os regulamentos, as estratégias e as ferramentas gerenciais precisariam estar em certa convergência com as aspirações individuais, ou mais precisamente, com a identidade do trabalhador, para que todos esses elementos possam fazer sentido em seu propósito maior. A literatura amparada na racionalidade instrumental defende que essa condição harmoniosa já foi alcançada, porém, a perspectiva da racionalidade substantiva pressupõe que isso ainda não é uma realidade (MUZZIO, 2012).

Independentemente da perspectiva epistemológica, a história organizacional mostra ações que significaram ganhos para as organizações nas áreas de produtividade, eficácia, competitividade e bem estar funcional. Para que a criatividade flua adequadamente em sistemas de colaboração coletiva, é preciso levar em consideração o conhecimento, os processos e o estilo de trabalho. Até mesmo o ambiente físico pode influenciar na capacidade criativa do indivíduo (MARTENS, 2011). O espaço simbólico também favorece o exercício da criatividade, tal como: horários mais flexíveis, atmosfera organizacional receptiva ao novo e tolerante com a possibilidade do erro na investida para uma solução criativa, modelos de gestão mais direcionados para a aprendizagem e sistemas de incentivos formais que motive o indivíduo criativo na geração de soluções inovadoras.

Estruturas de proteção em ambientes de refúgio com o processo inovador distanciado da rotina organizacional constituem espaços inovadores seguros, onde esse processo estaria alheio à realização de tarefas administrativas clássicas. Por outro lado, existe a possibilidade do risco de ambientes de refúgio se transformarem em torres de marfim, uma vez que o ato de disseminar e integrar novos conhecimentos e ideias à estruturas e práticas organizacionais cotidianas, de uma posição distante, representa uma tarefa quase impossível (CLEGG; KORNBERGER; PITSIS, 2011).

Além disso, a criatividade requer fatores ambientais de trabalho que propiciem o compartilhamento de informações, as controvérsias construtivas, a confiança, a participação da equipe no fluxo de ideias e nas relações abertas na equipe (MARTENS, 2011). Nessa lógica, existe a necessidade de a racionalização organizacional andar em paralelo com um ambiente criativo sem causar tensões nas dinâmicas desse novo mercado, buscandose um equilíbrio entre o imperativo da liberdade criativa e o imperativo comercial (LAMPEL; LANT; SHAMSIE, 2000).

Nessa linha de pensamento, uma suposição plausível é a possibilidade de 
uma maior convergência entre $\mathrm{o}$ desenvolvimento da criatividade a partir das características identitárias e o contexto propício disponibilizado pelas organizações. Para isso, uma ação efetiva para tal convergência seria oriunda principalmente das organizações, diante de sua capacidade institucional de influenciar nos parâmetros operacionais e simbólicos.

$\mathrm{Se}$ as ideias do indivíduo criativo não são implementadas ou não contribuem para novos produtos, serviços ou práticas gerenciais, sua criatividade deixa de ser aproveitada, dado que a geração de ideias e a efetivação da inovação representam fases distintas (SOUSA; PELLISSIER; MONTEIRO, 2012). Tal condição coloca o fator de convencimento e a capacidade de mobilizar suporte de apoio ao novo como condições significativas para a implantação das ideias criativas (BAER, 2012). Nessa ordem, a efetividade da ação criativa constitui um processo social de persuasão também influenciado pelas expectativas em torno do que o novo possa representar (YUAN; WOODMAN, 2010).

A literatura trata a questão da criatividade por diferentes aspectos, entretanto, um ponto em comum nesse debate reside na existência de um contexto capaz de favorecer o comportamento criativo e inovador. As características da organização, de seus membros e do seu ambiente, bem como os fatores estruturais do grupo - tais como, tempo de trabalho em conjunto, recursos disponíveis e qualidade das interações -, são exemplos de ações que podem ser valorizadas para alcançar uma convergência entre indivíduo e ambientes criativos.

Se admitirmos que essas ações são operacionalizadas por pessoas e são esses indivíduos que produzem uma condição superior das organizações (BARNEY, 1991), as políticas, as estratégias e os processos organizacionais deveriam buscar adequação à identidade individual e, sobretudo, à identidade no plano organizacional em seu viés legitimador (BROWN; TOYOKI, 2013), pois é nesse nível que o indivíduo estabelece as relações sociais e busca um equilíbrio entre o que ele deseja ser e o que o grupo espera que ele seja.

Enfim, uma organização não é criativa apenas por operar na indústria criativa. Por exemplo, uma organização burocrática e conservadora pode atuar no ramo do entretenimento, embora isso não a torne necessariamente criativa. Essa condição organizacional, portanto, está relacionada aos padrões culturais, relacionais e estruturais desenvolvidos em suas dependências e, nesse caso, a convergência entre os padrões administrativos organizacionais e a identidade do indivíduo criativo pode 
potencializar o uso da criatividade na dinâmica da inovação.

\section{CONSIDERAÇÕES FINAIS}

Para aqueles trabalhadores criativos que estão vinculados formalmente às organizações, são vários os desafios. Nas organizações em um sentido mais geral, o indivíduo é tolhido e precisa se ajustar a um esquema organizacional hostil ao processo libertário da criatividade. Pode-se admitir a existência de certa angústia do indivíduo criativo em decorrência do distanciamento de seu potencial e atitude criativos com a cultura normativa organizacional prevalecente. Como a criatividade está centrada no indivíduo em seu contexto coletivo, há uma incoerência entre ambientes burocráticos com culturas organizacionais não propícias à inovação e ambientes organizacionais necessários a uma fluidez criativa.

As organizações que atuam no universo da criatividade são um exemplo de onde essas novas condições seriam mais adequadas. Nesse sentindo, as estruturas, as regras, os processos e as culturas dessas organizações teriam mais coerência se propiciassem um ambiente que favorecesse a criatividade.

No novo formato de trabalho criativo, é evidente a manifestação de um tipo de neofordismo que assume o formato de uma produção direcionada para a superação das incertezas e instabilidades do mercado. Ao revelar características da especialização flexível, a nova ocupação criativa supõe a avidez por relações de trabalho que garantam a qualidade de vida do indivíduo que desenvolve seu empenho profissional em tarefas criativas.

A capacidade criativa do indivíduo está coadunada com sistemas de colaboração coletiva que contemplam o conhecimento, os processos inovadores de gestão, os estilos e formatos de trabalho mais flexíveis e o ambiente físico adaptado a uma atmosfera de incentivos à produção de perspectiva inovadora. A atividade criativa necessita, ainda, de certo nível de abertura a pessoas com ideias diferenciadas, de desembolsos financeiros sem retornos de curto prazo, de ambiência de compartilhamento, de intercâmbio de ideias, de controvérsias construtivas, de desafios constantes no trabalho, de confiança e de colaboração entre os membros das equipes.

A centralidade do indivíduo na economia criativa traz à tona a identidade como fator de ciência das peculiaridades organizacionais. Esse fato está entrelaçado a processos identitários de liberdade e autonomia exercidos pelo trabalhador criativo, os quais impõem a esse profissional a coexistência constante com os riscos de mercado e com a transferência para seus ombros dos gravames indiretos 
das precariedades do novo modo de produção.

Pode-se apontar, como um avanço no conhecimento, o desafio de ampliar o nível de cooperação entre indivíduos. Nesse sentido, o futuro parece levar a um contexto organizacional que seja permissivo a uma multiplicidade de identidades em que, paradoxalmente, as diversas identidades autônomas possam agir de maneira orquestrada. Por meio de uma analogia, pode-se inferir que o desafio de convivência intercultural, o qual facilitou a formação de equipes de diferentes culturas em prol dos resultados organizacionais e do respeito ao indivíduo diferente, precisa agora servir de inspiração para a coexistência das identidades pós-modernas autônomas, mas dependentes de uma legitimidade social, bem como, de um compartilhamento de tarefas com outros indivíduos igualmente autônomos e distintos na identidade, no complexo sistema de inovação e na criatividade que se faz realidade.

Como proposição para a consolidação de uma gestão de organizações criativas que seja alinhada com as características identitárias do indivíduo criativo, defende-se que as práticas organizacionais, capitaneadas pelas lideranças, poderiam agir em distintos níveis: As ações de liderança poderiam caminhar para que o indivíduo reconheça sua centralidade no processo criativo e para que instrumentalize suas características identitárias de inquietação a fim de alavancar no processo de inovação com ganhos individuais e coletivos. Ainda, as lideranças poderiam agir para que o grupo possa ser um elemento legitimador e mesmo incentivador de uma postura de autonomia identitária de criação e transformação que se reverteria em ganhos coletivos. Isso pode ser executado pela constituição de rede de grupos interdisciplinares para prover soluções criativas que se constituam respostas aos desafios organizacionais. Por fim, os líderes poderiam fomentar um entendimento da coletividade que venha a contemplar a identidade criativa como um valor da cultura da organização, podendo assim, ser transmitido e disseminado aos membros como algo adequado e que deve ser perpetuado para o bem da coletividade. Isso pode ser executado, por exemplo, pela introdução do tema criatividade em normas, na inclusão da postura criativa, como um valor, e nas manifestações simbólicas, como os rituais de premiação ou promoção.

No futuro, novas pesquisas podem averiguar as percepções e condições de identidade prevalecentes entre indivíduos criativos e disseminar o que esses agentes esperam de suas lideranças, bem como de ajustes em regras sociais e operacionais 
vigentes em suas organizações, inclusive com foco nas relações entre interagentes e sua influência no processo criativo. Pesquisas podem ainda identificar variáveis que influenciam no processo de criatividade gerencial. Outros estudos podem ainda vislumbrar modos de como as práticas das organizações contemporâneas de perfil criativo são redefinidas em comparação com modelos clássicos de gestão, tais exemplos tenderiam a revelar a existência de padrões de ação que ajudariam a aclarar o conhecimento a respeito das condições diferenciadas dessas organizações.

A contribuição do estudo reside em ter trazido a abordagem da economia criativa como ótica temática de análise das tensões e mudanças constantes dotadas de significados que contemplam o universo dos profissionais hipercriativos e suas identidades. Isso representa um passo além do reducionismo das determinações organizacionais de estrutura clássica com leituras dos estudos organizacionais pautadas tão somente pela lógica das relações de trabalho que dispensam o olhar crítico sob o prisma do componente identitário do ator criativo.

\section{REFERÊNCIAS}

ALVESSON, M. Self-doubters, strugglers, storytellers, surfers and others: Images of self-identities in organization studies.
Human Relations, v. 63, n. 2, p. 193-217, 2010 .

ALVAREZ, M. D. Creative cities and cultural spaces: new perspectives for city tourism. International Journal of Culture, Tourism and Hospitality Research, v. 4, n. 3, p. 171-175, 2010.

ANDERSON, N.; POTOČNIK, K.; ZHOU, J. Innovation and Creativity in Organizations: A State-of-the-Science Review, Prospective Commentary, and Guiding Framework. Journal of Management, v. 40, n. 5, p. 1297-1333, 2014.

BACH. L. Exploring the convergence of the rationales underlying the policies for science, technology and innovation and for creative industries. In BURGERHELMCHEN, T. Economics of Creativity: ideas, firms and markets. Oxon, New York: Routledge, 2013.

BAER, M. Putting Creativity to Work: The Implementation of Creative Ideas in Organizations. Academy of Management Journal, v. 55, n.5, p. 1102-1119, 2012.

BARNEY, J. Firm resources and sustained competitive advantage. Journal of Management, v. 17, n. 1, p. $99-120$, 1991.

BENDASSOLLI, P. F.; BORGESANDRADE, J. E. Significado do Trabalho nas Indústrias Criativas. Revista de Administração de Empresas, v. 51, n.2, p. 143-159, 2011.

BOON, B.; JONES, D.; CURNOW, B. Out of the Blue: the dark side of creative enterprise. Culture and Organization, v. 15, n. 3-4, p. 361-377, 2009.

BROWN, A. D.; TOYOKI, S. Identity Work and Legitimacy. Organization Studies, v. 34, n. 7, p. 875-896, 2013. 
BURGEN-HELMCHEN, T. Economics of Creativity: 0. Oxon, New York: Routledge, 2013.

CHASTON， I.; SADLER-SMITH， E. Entrepreneurial Cognition, Entrepreneurial Orientation and Firm Capability in the Creative Industries. British Journal of Management, v. 23, n. 3, p. 415-432, 2012.

CHREIM, S.; WILLIAMS, B. E. B.; HININGS, C. R. B. Inter level Influences on the Reconstruction of Professional Role Identity. Academy of Management Journal, v. 50, n. 6, p. 1515-1539, 2007.

CHRISTOPHERSON, S. Beyond the Selfexpressive Creative Worker: an Industry Perspective on Entertainment Media. Theory, Culture \& Society, v. 25, n. 7-8, p. 73-95, 2008.

CLARKE, C. A.; BROWN A. D.; HAILEY, V. H. Working identities? Antagonistic discursive resources and managerial identity. Human Relations, v. 62, n. 3, p. 323-352, 2009.

CLEGG, S.; KORNBERGER, M.; PITSIS, T. Administração e Organizações: uma introdução à teoria e a prática. 2. Ed. Porto Alegre: Bookman, 2011.

CORLEY, K. G.; GIOIA, D. A. Identity Ambiguity and Change in the Wake of a Corporate Spin-off. Administrative Science Quarterly, v. 49, n. 2, p. 173208, 2004.

COSTA, J. H. Inovação organizacional, flexibilização e precarização: dilemas da "qualidade de vida no trabalho".

Contribuciones a las ciências sociales, p.1-14, 2015.

DAVIS, C. H.; CREUTZBERG, T.; ARTHURS, D. Applying an innovation cluster framework to a creative industry: The case of screen-based media in Ontario.
Innovation: Management, Policy \& Practice, v. 11, n. 2, p. 201-214, 2009.

DEFILLIPPI, R.; GRABHER, G.; JONES, C. Introduction to paradoxes of creativity: managerial and organizational challenges in the cultural economy. Journal of Organizational Behavior, v. 28, n. 5, p. 511-521, 2007.

DOWN, S.; REVELEY, J. Between narration and interaction: Situating firstline supervisor identity work. Human Relations, v. 62, n.3, p. 379-401, 2009.

FLORIDA, R. A Ascensão da classe criativa: e seu papel na transformação do trabalho, do lazer, da comunidade e do cotidiano. Porto Alegre: LPM, 2011.

GIBSON, C.; LUCKMAN, S.; WILLOUGHBYSMITH, J. Creativity without Borders? Rethinking remoteness and proximity. Australian Geographer, v. 41, n. 1, p. 25-38, 2010.

GILL R.; PRATT A. Precarity and Cultural Work in the Social Factory? Immaterial Labour, Precariousness and Cultural Work. Theory, Culture \& Society, v. 25, n. 7-8, p. 1-30, 2008.

GOEDE, M.; LOUISA, G. A case study of the creative zone Scharloo and Pietermaai in Curaçao. International Journal of Social Economics, v. 39, n. 11, p. 844 $858,2012$.

GUERRA, J. R. F.; PAIVA JR., F. G. A. Produção Empreendedora Cultural de Pernambuco: O caso do audiovisual pela REC Produtores Associados. In MACHADO, H. V. Empreendedorismo, Oportunidades e Cultura. Maringá: UEM, 2013.

HARDT, M.; NEGRI, A. Império. 10 Ed. Rio de Janeiro: Record, 2012.

LAMPEL, J.; LANT, T.; SHAMSIE, J. Balancing act: Learning from organizing 
practices in cultural industries.

Organization Science, v. 11, n. 3, p. $263-$ 269, 2000.

LOBATO, R. Creative industries and informal economies: lessons from Nollywood. International Journal of Cultural Studies, v. 13 , n.4, p. 337-354, 2010.

MACHADO, H. V. A Identidade e o Contexto Organizacional: Perspectivas de Análise. Revista de Administração Contemporânea, Edição Especial, p.5173, 2003.

MARTENS, Y. Creative workplace: instrumental and symbolic support for creativity. Facilities, v. 29, n. 1, p. 63-79, 2011.

MUZZIO, H. Racionalidade em jogo em um processo de profissionalização organizacional. Revista de Administração Contemporânea, v. 16 , n. 6 , p. 827-844, 2012.

O'CONNOR, J.; GU, X. Developing a Creative Cluster in a Postindustrial City: CIDS and Manchester. The Information Society, v. 26, n. 2, p. 124-136, 2010.

PEUTER, G. de. Creative Economy and Labor Precarity: A Contested Convergence. Journal of Communication Inquiry, v. 35, n. 4, p. 417-425, 2011.

ROWLANDS, L.; HANDY, J. An addictive environment: New Zealand film production workers' subjective experiences of project-based labour. Human Relations, v. 65, n. 5, p. 657-680, 2012.

SAAKI, M. Urban Regeneration through Cultural Creativity and Social Inclusion: Rethinking Creative City Theory through a Japanese Case Study. Cities, v. 27, 2010.

SIEBERT, S.; WILSON, F. All work and no pay: consequences of unpaid work in the creative industries. Work
Employment Society, v. 27, n. 4, p. 711$721,2013$.

SONGJIE, L.; XINGHUA, L. The Role of Cultural Creative Industry in the City Development: The Case of Jingdezhen. Studies on Sociology of Science, v. 2, n. 2, p. 74-78, 2011.

SOUSA, F. C. De; PELLISSIER, R.; MONTEIRO, I. P. Creativity, Innovation and Collaborative Organizations. The International Journal of Organization Innovation, v. 5, n. 1, p. 26-64, 2012.

TENÓRIO, F. G. A unidade dos contrários: fordismo e pós-fordismo. Revista de Administração Pública, v. 45, n. 4, p. 1141-1172, 2011.

\section{UNITED NATIONS CONFERENCE ON TRADE AND DEVELOPMENT. Economia Criativa: Uma Opção de Desenvolvimento Viável. São Paulo, 2010.}

WANG, A.; CHENG, B. When does benevolent leadership lead to creativity? The moderating role of creative role identity and job autonomy. Journal of Organizational Behavior, v. 31, n. 1, p. 106-121, 2010.

YBEMA S, et al. Articulating identities. Human Relations, v. 62, n. 3, p. 299-322, 2009.

YUAN, F.; WOODMAN, R. W. Innovative behavior in the workplace: The role of performance and image outcome expectations. Academy of Management Journal, v. 53, n. 2, p. 323-342, 2010. 